\begin{tabular}{|c|l|}
\hline Title & Identification of genetic loci affecting the establishment and development of Echinococcus multilocularis larvae in mice \\
\hline Author(s) & $\begin{array}{l}\text { Nakao, Ryo; Kameda, Yayoi; Kouguchi, Hirokazu; Matsumoto, Jun; Dang, Zhisheng; Simon, Ayo Y ila; Torigoe, } \\
\text { Daisuke; Sasaki, Nobuya; Oku, Y uzaburo; Sugimoto, Chiriro; A gui, Takashi; Y agi, Kinpei }\end{array}$ \\
\hline Citation & $\begin{array}{l}\text { International Journal for Parasitology, 41(11), 1121-1128 } \\
\text { https://doi.org/10.1016/.ijpara.2011.06.007 }\end{array}$ \\
\hline Issue Date & 2011-09 \\
\hline Doc URL & http://hdl.handle.net/2115/51381 \\
\hline Type & article(author version) \\
\hline File Information & IJP41_1121.pdf \\
\hline
\end{tabular}

Instructions for use 


\section{Identification of genetic loci affecting the establishment and development of Echinococcus}

\section{multilocularis larvae in mice}

Ryo Nakao ${ }^{\text {a, }}$, Yayoi Kameda ${ }^{\text {b, }}$, Hirokazu Kouguchi ${ }^{\mathrm{c}}$, Jun Matsumoto ${ }^{\mathrm{d}}$, Zhisheng Dang ${ }^{\mathrm{a}, \mathrm{e}}$, Ayo Yila Simon ${ }^{\mathrm{b}}$, Daisuke Torigoe ${ }^{\mathrm{b}}$, Nobuya Sasaki ${ }^{\mathrm{b}}$, Yuzaburo Oku ${ }^{\mathrm{e}}$, Chihiro Sugimoto ${ }^{\mathrm{a}}$, Takashi Agui ${ }^{\mathrm{b}}$, Kinpei Yagi ${ }^{*}$

${ }^{a}$ Department of Collaboration and Education, Research Center for Zoonosis Control, Hokkaido University, Kita 20, Nishi 10, Kita-ku, Sapporo, Hokkaido 001-0020, Japan

${ }^{\mathrm{b}}$ Laboratory of Laboratory Animal Science and Medicine, Department of Disease Control, Graduate School of Veterinary Medicine, Hokkaido University, Kita 18, Nishi 9, Kita-ku, Sapporo, Hokkaido 060-0818, Japan

${ }^{\mathrm{c}}$ Department of Biological Science, Hokkaido Institute of Public Health, Kita 19, Nishi 12, Kitaku, Sapporo, Hokkaido 060-0819, Japan

${ }^{\mathrm{d}}$ Laboratory of Medical Zoology, Department of Veterinary Medicine, Nihon University College of Bioresource Sciences, 1866 Kameino, Fujisawa, Kanagawa 252-0880, Japan

${ }^{\mathrm{e}}$ Department of Parasitology, School of Veterinary Medicine, Faculty of Agriculture, Tottori University, 4-101 Koyama, Tottori 680-8553, Japan

${ }^{1}$ These authors contributed equally to this work.

*Corresponding author. Tel.: +81 11747 0123; fax: +81 117369476.

E-mail address: kinpei@iph.pref.hokkaido.jp 


\section{ABSTRACT}

Alveolar echinococcosis (AE) is a severe hepatic disorder caused by larval infection by the fox tapeworm Echinococcus multilocularis. The course of parasitic development and host reactions are known to vary significantly among host species, and even among different inbred strains of mice. As reported previously, after oral administration of parasite eggs, DBA/2 (D2) mice showed a higher rate of cyst establishment and more advanced protoscolex development in the liver than C57BL/6 (B6) mice. These findings strongly suggest that the outcome of $\mathrm{AE}$ is affected by host genetic factor(s). In the present study, the genetic basis of such strain-specific differences in susceptibiliy/resistance to $\mathrm{AE}$ in murine models was studied by whole-genome scanning for quantitative trait loci (QTLs) using a backcross of $(\mathrm{B} 6 \times \mathrm{D} 2) \mathrm{F}_{1}$ and $\mathrm{D} 2$ mice with varying susceptibility to E. multilocularis infection. For cyst establishment, genome linkage analysis identified one suggestive and one significant QTL on chromosomes (Chrs.) 9 and 6, respectively, whereas for protoscolex development, two suggestive and one highly significant QTLs were detected on Chrs. 6, 17 and 1, respectively. Our QTL analyses using murine AE models revealed that multiple genetic factors regulated host susceptibility/resistance to $E$. multilocularis infection. Moreover, our findings show that establishment of the parasite cysts in the liver is affected by QTLs that are distinct from those associated with the subsequent protoscolex development of the parasite, indicating that different host factors are involved in the 
host-parasite interplay at each developmental stage of the larval parasite. Further identification of responsible genes located on the identified QTLs could lead to the development of effective disease prevention and control strategies, including an intensive screening and clinical follow-up of genetically high-risk groups for AE infection.

Keywords: Echinococcus multilocularis, Alveolar echinococcosis, Zoonosis, QTL, Cyst, Protoscolex, Emcys1, Empsc1 


\section{Introduction}

Alveolar echinococcosis (AE) is a severe hepatic disorder caused by the metacestode stage of the tapeworm Echinococcus multilocularis. This zoonotic disease is endemic in many regions of the northern hemisphere and is one of the most significant life-threatening helminth diseases (Craig et al., 1996). The intermediate hosts, mainly rodents and occasionally humans, become infected by oral uptake of the eggs excreted in the feces of final host carnivores. The larval oncospheres hatch from eggs in the intestine, most probably penetrate the intestinal wall and disseminate primarily into the liver. The larvae establish in the liver and develop to harbor mature protoscoleces.

Many researchers have used inbred mice as experimental models of AE to study the hostparasite interplay. However, the course of parasitic development and host reactions are known to vary significantly among mouse strains (Yamashita et al., 1958; Webster and Cameron, 1961; Gottstein and Felleisen, 1995; Bauder et al., 1999; Hildreth and Granholm, 2003; Matsumoto et al., 2010). Previous study showed that the two inbred strains, DBA/2 (D2) and C57BL/6 (B6), differ markedly in their susceptibility to E. multilocularis infection (Matsumoto et al., 2010).

When infective eggs were administered orally, D2 mice had a higher number of cysts established in the liver than did B6 mice, and moreover, mature protoscoleces were observed only in D2 
mice at 16 weeks p.i.. Although this observation supported the belief that the susceptibility to $E$. multilocularis infection is genetically controlled, as has been previously suggested for animals and humans (Nakaya et al., 1997; Hildreth and Granholm, 2003; Vuitton, 2003), genetic factors responsible for the differences in susceptibility remain unknown.

Quantitative trait locus (QTL) mapping is a promising tool for the detection of genetic loci that contribute to differences in phenotypic variation. In this study, the traits and genotypes of offspring derived from two different strains of animals were investigated on a genome-wide scale to determine statistical linkages and to identify loci which contained genes affecting the traits. To date, the genetic background of susceptibility to nematode infection has been investigated using this approach in animals (Iraqi et al., 2003; Suzuki et al., 2006; Beraldi et al., 2007) as well as in humans (Williams-Blangero et al., 2008).

In this study, QTL analysis was conducted on offspring derived from D2 and B6 mice with varying susceptibility to E. multilocularis infection. Genetic linkage analysis identified one significant and one highly significant QTL for the establishment and development of $E$. multilocularis larvae in mice. Furthermore, our data show that establishment and development of parasites are controlled by distinct and multiple genetic factors. 


\section{Materials and methods}

2.1. Mice

D2, B6 and $(B 6 \times D 2) F_{1}\left(F_{1}\right)$ mice were purchased from Japan SLC (Shizuoka, Japan). $F_{1}$

$\times$ D2 backcross $\left(\mathrm{N}_{2}\right)$ mice were generated to conduct the genetic and phenotypic assessment

experiments. All animal experiments were performed in accordance with the guidelines of

Hokkaido Institute of Public Health, Japan and were approved by the ethics committee of the Institute.

\subsection{Experimental infection}

Echinococcus multilocularis eggs were obtained from the feces of two beagle dogs that had been orally inoculated with mature metacestode tissue containing protoscoleces (Hokkaido isolate). The eggs were then washed several times with physiological saline and kept at $4^{\circ} \mathrm{C}$ until use for experimental infections in mice. The experimental infections were performed in three independent and identical trials using these infective eggs, prepared 1-3 months prior to each experimental infection. The anesthetized mice, aged 4-5 weeks, were administered with either 
200 or 2,000 eggs suspended in $0.5 \mathrm{ml}$ of physiological saline orally. The animals were handled within a safety facility (Biosafety Level 3) at the Hokkaido Institute of Public Health, Japan.

\subsection{Examination of cyst establishment and protoscolex development}

A total of 148 mice (D2 $\left.(n=10) ; \mathrm{B} 6(n=10) ; \mathrm{F}_{1}(n=10) ; \mathrm{N}_{2}(n=118)\right)$ infected with 200 eggs were sacrificed and necropsies were performed at 4 weeks p.i. for the assessment of cyst establishment. The livers were cut into approximately $0.5 \mathrm{~mm}$-thick slices and the total numbers of cysts were counted. A total of 283 mice (D2 $(n=11) ; \mathrm{B} 6(n=10) ; \mathrm{F}_{1}(n=12) ; \mathrm{N}_{2}(n$ $=250))$ infected with 2,000 eggs were sacrificed and necropsies were performed at 16 weeks p.i.

for the assessment of protoscolex development. The livers were collected and the total weight of the organs and that of host liver tissue and parasitic cysts were measured. Several parts of the cysts (total weight $1-2 \mathrm{~g}$ ) were minced, passed through a $125 \mu \mathrm{m}$ sieve, and then washed repeatedly with physiological saline. The numbers of mature protoscoleces were counted under a stereoscopic microscope as an index of the parasite development. The number of eggs used for the first experiment $(n=200)$ was expected to produce a macroscopically countable number of cystic lesions in the host liver, while the number used in the second experiment $(n=2,000)$ was expected to reduce the effect of the difference in the numbers of cysts established in individual mice. 


\subsection{Genotype analysis}

To identify and map QTLs, a total of 121 informative microsatellite markers-113 for cyst establishment and 117 for protoscolex development-were chosen from a database maintained by Mouse Genome Informatics (MGI), The Jackson Laboratory, USA (http://www.informatics.jax.org/), as listed in Table 1. After the initial QTL scan, extra markers were included in the vicinity of the provisional QTLs to refine their position. A total of $232 \mathrm{~N}_{2}$ mice, 118 for cyst establishment and 114 for protoscolex development, were genotyped using each microsatellite marker. After genomic DNA extraction and PCR amplification, amplified PCR products were electrophoresed in $10 \%$ polyacrylamide or $3 \%$ agarose gels, stained with ethidium bromide and visualized under UV light. The genotype at each marker locus was determined by the size of the PCR product.

\subsection{Data analysis}

Two phenotypes - cyst establishment and protoscolex development in mice-were analyzed separately and the following phenotypic parameters were used for the analyses. For the analysis of cyst establishment, the number of cysts in the liver was converted to a percent value against a mean number of cysts in susceptible D2 mice to avoid the bias resulting from egg infectivity between two independent experimental infections. For the analysis of protoscolex 
development, the number of mature protoscoleces in the liver was indicated in terms of number/gram of cystic lesion, which was subsequently log-transformed for QTL analysis. Zero values were assigned a value of 1 to allow log transformation. Linkage analyses of two phenotypes to chromosomal loci were performed using the MapManager QTXb20 software (Manly et al., 2001). Recombination frequencies (\%) were converted into genetic distances (centiMorgan; cM) using the Kosambi map function, in which linkage data are provided as likelihood ratio statistic (LRS) scores. Genome-wide significance thresholds were calculated in terms of LRS by carrying out permutation tests for 1,000 permutations based on the established guidelines (Lander and Kruglyak, 1995). The thresholds in the backcrossed progenies were determined by the QTL software program for finding suggestive, significant and highly significant linkages. Two-way interactions (epistasis) were estimated with a QTL scan and statistical significance for these gene to gene interaction tests were based on $P<10^{-5}$ using 1,000 permutations of the observed data. Statistical analysis was performed using the Stat-View program (SAS Institute, Cary, NC, USA). $P<0.05$ was considered to be significant. A Scheffé's F test was conducted for multiple comparisons between the mean values of genotypes at the detected QTLs. 


\section{Results}

\subsection{Establishment of E. multilocularis cysts}

At 4 weeks after oral administration of 200 eggs, the total numbers of cysts in the liver were counted. In two independent experiments, the average numbers of cysts in D2 mice were different (average \pm S.D.): $79.0 \pm 30.4$ and $60.6 \pm 13.5$ for the first and second experiments, respectively. Therefore, the numbers of cysts observed in B6, $\mathrm{F}_{1}$ and $\mathrm{N}_{2}$ mice were converted to percent values against a mean number of cysts in susceptible D2 mice in each experiment. The distribution of percent values are shown in Fig. 1A. All animals had cestodes in the livers. The mean values for B6 mice $(40.2 \%)$ were significantly lower than those of D2 mice $(P<0.01$, Scheffé's $\mathrm{F}$ test), while those of $\mathrm{N}_{2}$ progenies varied from 1.7 to $182.5 \%$ with an average of $72.0 \%$.

\subsection{Identification of QTLs and their effects on cyst establishment}

The genome-wide linkage analysis of cyst establishment was performed using $118 \mathrm{~N}_{2}$ mice, where one suggestive and one significant QTL were detected on chromosomes (Chrs.) 9 and 6 (Emcys1), respectively (Figs. 2A and 3A). The significant QTL had a peak LRS score of 15.0 and accounted for $12 \%$ of the total variance (Table 2). No significant epistatic interactions were observed between all of the tested markers (data not shown). Segregation of $\mathrm{N}_{2}$ mice according to their genotypes at Emcys (D6Mit150) showed that individuals homozygous for the 
D2 allele (DD) had a statistically higher number of cysts than did the heterozygous mice (DB) $(P$ $<0.01$, Scheffé 's F test), and that there were no significant differences between D2 and DD, and between B6, $\mathrm{F}_{1}$ and DB (Fig. 4A). A list of Emcys 1 candidates on Chr. 6 is shown in Table 3.

\subsection{Development of E. multilocularis protoscoleces}

At 16 weeks after oral administration of 2,000 eggs, the total numbers of mature protoscoleces in the liver were calculated. The distributions of numbers of mature protoscoleces per gram of cystic tissue are shown in Fig. 1B. Although all animals had Echinococcus cysts in their enlarged livers, the number of mature protoscoleces differed greatly among individual mice even within the same mouse group. In D2 mice, $1.5 \times 10^{4}-8.5 \times 10^{5}$ protoscoleces were recovered per gram of cystic lesion, whereas in all $\mathrm{B} 6$ mice and some $\mathrm{N}_{2}$ progenies, protoscolex formation was completely absent (Fig. 1B).

\subsection{Identification of QTLs and their effects on protoscolex development}

The genome-wide linkage analysis of protoscolex development using $114 \mathrm{~N}_{2}$ mice identified two suggestive and one highly significant QTLs on Chrs. 6, 17 and 1 (Empsc1), respectively (Figs. 2B and 3B). The highly significant QTL had a peak LRS score of 75.4 and accounted for $48 \%$ of total variants (Table 2). The two suggestive QTLs with LRS scores of 7.1 and 10.3 explained only 6 and $9 \%$ of the variants, respectively. Although weak epistatic interaction was observed between Empsc1 and suggestive QTLs, the scores did not reach the 
significant level (data not shown). $\mathrm{N}_{2}$ carrying D2 mice homozygous at Empsc1 (D1Mit14) had statistically higher numbers of mature protoscoleces than did DB heterozygous mice $(P<0.001$, Scheffé 's F test), and there were no significant differences between D2 and DD and between $F_{1}$ and DB (Fig. 4B). A list of Empsc1 candidates on Chr. 1 is shown in Table 3.

\section{Discussion}

As shown in a previous investigation, susceptibility/resistance to E. multilocularis infection was different among mouse strains including D2 and B6 mice (Matsumoto et al., 2010). D2 mice were more susceptible to infection than were B6 mice. D2 mice showed a higher number of cysts established in the liver after administration of infective eggs than those of B6, and at 16 weeks p.i., maturation of protoscoleces occurred only in D2, but not in B6 mice. The objective of this study was to perform a genome-wide linkage analysis of a cross between D2 and B6 mice to localize genes associated with the increased susceptibility of D2 mice against $E$. multilocularis infection.

We found significant linkage between cyst establishment and genotypes at Emcys 1 on Chr. 6 with a peak LRS score of 15.0, accounting for $12 \%$ of the variation (Table 2). The segregation analysis supported a strong influence of Emcysl on cyst establishment (Fig. 4A).

However, the continuous distribution of $\mathrm{N}_{2}$ phenotypes implies that multiple genes control 
susceptibility to E. multilocularis infection. Although statistical modeling of the current data did not detect any QTL interacting with Emcys1, the suggestive QTL on Chr. 9, and even others slightly below the suggestive level (LRS = 6.7) such as those on Chrs. 3, 13 and 17 (Fig. 2A), may have an influence on susceptibility. At this time, it is difficult to identify a gene responsible for cyst establishment on Emcys1, a region between D6Mit188 and D6Mit15, as there are nearly 1,000 genes located within this chromosomal region. Even with the aid of the web-based program Positional Medline (PosMed; http://omicspace.riken.jp/PosMed) (Yoshida et al., 2009), approximately 200 immune-related genes that may be involved in host-parasite interplay, were identified, some of which are listed in Table 3.

The safety requirements for handling infective E. multilocularis eggs hinder the research on cyst establishment after oral infection with eggs and thus only limited information is available for further discussion on candidate genes. Gottstein et al. (2010) used microarray analysis to compare the expression levels of hepatic genes in mice between pre- and 31-days p.i. of eggs. The study identified only 38 genes whose expression levels were significantly changed. None of those were located on Emcys1, implying that the host factors, apart from those expressed in the liver, may have more influence on the cyst establishment and that the earlier stages of infection such as hatching, activation, penetration, migration and predilection of oncospheres might be determinants of susceptibility to the infection. As recently reviewed by Vuitton and Gottstein 
(2010), the cell-mediated immune responses, especially acute inflammatory Th1 response, are known to play an important role in the early stage of E. multilocularis infection. Furthermore, early inflammation induced by complement activation was shown to be important in controlling the establishment of the metacestode of Echinococcus granulosus, a closely related parasite (Breijo et al., 2008). The Emcys 1 locus includes genes related with the Th1 response, such as $C d 4, \operatorname{Lag} 3$ and $C d 69$, and those associated with complement components, such as $C 1 s, C 1 r, C l r l$ and C3arl (Table 3).

In the Chinese village of Nanwan, a highly endemic area for $\mathrm{AE}$, only certain family members were likely to be more susceptible to $\mathrm{AE}$ than others, despite sharing similar life patterns (Yang et al., 2006), implying that host genetic factors contribute to the susceptibility to E. multilocularis infection in humans. Some genes showing quantitative genetic variation in mice have also been revealed to affect the phenotypic variance in humans (Korstanje et al., 2004; Hillebrandt et al., 2005; Peters et al., 2007). Therefore, the data obtained in this study could provide an experimental basis for further identification of human genetic factors that are associated with the response after ingestion of E. multilocularis eggs. Such information is expected to facilitate the development of effective disease prevention and control programs, for example, an intensive screening of genetically high-risk groups for $\mathrm{AE}$ infection. 
The identification of genetic factors affecting the protoscolex development of $E$. multilocularis will lead to a better understanding of host and parasite interplay in the intermediate hosts. In our analysis, highly significant linkage was observed between protoscolex development and genotypes at Empsc1 on Chr. 1 (a peak LRS score of 75.4) (Fig. 3B). A significant influence of Empscl on variants was demonstrated by a high value of contribution (48\%) (Table 2) as well as significant differences between DD homozygous and DB heterozygous in segregation analysis (Fig. 4B), whereas the detection of other suggestive QTLs suggests that the protoscolex development is under multigenic control, as in the case of cyst establishment.

The physical barrier between growing parasites and host tissue is the laminated layer, an acellular and carbohydrate-rich outer membrane of cysts, which protect parasites from direct contact with host immune cells. Meanwhile, several host-derived molecules were previously reported to exist in the cyst fluid or on the wall (Kassis and Tanner, 1977; Ali-Khan and Siboo, 1981), indicating that certain host-derived factors are transported through the laminated layer and are involved in parasite development in the hosts. In recent years, great progress has been made in elucidating the mechanisms of parasite development in the hosts by identifying the evolutionarily conserved signaling systems in E. multilocularis, which can interact with hostderived molecules (Konrad et al., 2003; Spiliotis et al., 2003, 2005, 2006; Zavala-Góngora et al., 
2003, 2006, 2008; Gelmedin et al., 2008, 2010; Brehm, 2010; Förster et al., 2011). Specifically, interactions between parasite receptors and host-derived molecules, such as epidermal growth factor and insulin have been demonstrated experimentally (Spiliotis et al., 2006; Gelmedin et al., 2008; Brehm, 2010), strongly suggesting that parasite development is triggered by host signaling systems. Of 450 genes located on the Empscl locus, more than 100 were previously associated with various kinds of signaling systems in mammals, as exemplified in Table 3. Thus, a better understanding of evolutionarily conserved E. multilocularis signaling systems may lead to further identification of host factors important for parasite development from the existing list of candidates.

In humans, major histocompatibility complex (MHC) genotypes were associated with the clinical severity of AE (Eiermann et al., 1998; Godot et al., 2000; Zhang et al., 2003). In mice, the genes coding for MHC molecules are located on Chr. 17 and thus are not included in the Empsc1 locus determined in this study. In our experimental infections of mice, there was no statistically significant correlation between the number of mature protoscoleces and whole liver weight at 16 weeks p.i. measured as an index of metacestode growth in size (data not shown). These findings suggest that the metacestode growth of E. multilocularis is regulated, at least in part, by host factors different from those affecting protoscolex development. As generally recognized, mature protoscoleces are critical for the parasite to maintain its lifecycle, whereas 
the growth of the metacestode mass is intimately associated with the clinical outcome of AE.

Hence, further investigations to identify QTLs responsible for metacestode growth in murine models may experimentally demonstrate the association between MHC genotypes and disease severity that was suggested in AE cases.

The database available at the MGI website (http://www.informatics.jax.org/strains_SNPs.shtml) provides information on the strain-specific genomic features of mice. In the present investigation, however, only limited numbers of genomic differences were found between D2 and B6 strains on the identified QTLs (nine and 18 for Emcysl and Empsc1, respectively), all of which were located outside the coding region (data not shown). Since the database will be extended regularly, it might become useful to further narrow down the candidate genes in the future.

Using congenic techniques, we are currently attempting to generate mice strains that harbor QTLs from one selection line on the opposite line to investigate whether each allele has a different effect on the phenotype. At the same time, several approaches are currently being employed to identify candidate genes located on Emcys 1 and Empsc1. Some of these approaches include comparisons of gene expression levels of D2 and B6 mice in response to $E$. multilocularis infection using microarray and next-generation RNA sequencing technologies. This analytical combination that includes QTL mapping and gene expression profiles has proven 
useful in the selection of candidate genes (Wayne and McIntyre, 2002; Rennie et al., 2008; Ahn

et al., 2010; Stark et al., 2010).

A lack of existing comprehensive information on the interplay between parasite and intermediate host makes it difficult to focus on certain genes responsible for resistance/susceptibility to E. multilocularis infection. However, our study provides several important conclusions. First, using QTL analysis, we were able to localize chromosomal sites where the allelic differences in genes present in D2 and B6 mice strongly affect mouse susceptibility to E. multilocularis infection. Second, multiple QTLs were detected on different Chrs., indicating that susceptibility to infection is complex and is determined by multiple host genes. Additionally, larval establishment and development in mouse livers are controlled by distinct QTLs, indicating that there are different host factors interplaying with parasites at each developmental stage. Further identification of responsible genes located on the identified QTLs could lead to the development of effective disease prevention and control strategies, for example, an intensive screening and clinical follow-up of genetically high-risk groups for AE infection.

\section{Acknowledgements}

The first author was supported by a research grant fellowship from the Japanese Society

for the Promotion of Science (JSPS) for young scientists. This work was financially supported by 
the Ministry of Education, Culture, Sports, Science and Technology of Japan (MEXT) Grant-inAid for Scientific research (B) \#20380164, the Global Center of Excellence (COE) Program, "Establishment of International Collaboration Centers for Zoonosis Control" from MEXT, and the Academic Frontier Project for Private Universities from MEXT. 


\section{References}

Ahn, S.H., Deshmukh, H., Johnson, N., Cowell, L.G., Rude, T.H., Scott, W.K., Nelson, C.L.,

Zaas, A.K., Marchuk, D.A., Keum, S., Lamlertthon, S., Sharma-Kuinkel, B.K., Sempowski,

G.D., Fowler, V.G., 2010. Two genes on A/J chromosome 18 are associated with

susceptibility to Staphylococcus aureus infection by combined microarray and QTL

analyses. PLoS Pathog. 6, e1001088.

Ali-Khan, Z., Siboo, R., 1981. Echinococcus multilocularis: distribution and persistence of specific host immunoglobulins on cysts membranes. Exp. Parasitol. 51, 159-168.

Bauder, B., Auer, H., Schilcher, F., Gabler, C., Romig, T., Bilger, B., Aspöck, H., 1999.

Experimental investigations on the $\mathrm{B}$ and $\mathrm{T}$ cell immune response in primary alveolar echinococcosis. Parasite Immunol. 21, 409-421.

Beraldi, D., McRae, A.F., Gratten, J., Pilkington, J.G., Slate, J., Visscher, P.M., Pemberton, J.M., 2007. Quantitative trait loci (QTL) mapping of resistance to strongyles and coccidia in the free-living Soay sheep (Ovis aries). Int. J. Parasitol. 37, 121-129.

Brehm, K., 2010. The role of evolutionarily conserved signalling systems in Echinococcus multilocularis development and host-parasite interaction. Med. Microbiol. Immunol. 199, 247-259. 
Breijo, M., Anesetti, G., Martínez, L., Sim, R.B., Ferreira, A.M., 2008. Echinococcus

granulosus: the establishment of the metacestode is associated with control of complementmediated early inflammation. Exp. Parasitol. 118, 188-196.

Craig, P.S., Rogan, M.T., Allan, J.C., 1996. Detection, screening and community epidemiology of taeniid cestode zoonoses: cystic echinococcosis, alveolar echinococcosis and neurocysticercosis. Adv. Parasitol. 38, 169-250.

Eiermann, T.H., Bettens, F., Tiberghien, P., Schmitz, K., Beurton, I., Bresson-Hadni, S., Ammann, R.W., Goldmann, S.F., Vuitton, D.A., Gottstein, B., Kern, P., 1998. HLA and alveolar echinococcosis. Tissue Antigens 52, 124-129.

Förster, S., Günthel, D., Kiss, F., Brehm, K., 2011. Molecular characterization of a serumresponsive, DAF-12-like nuclear hormone receptor of the fox-tapeworm Echinococcus multilocularis. J. Cell Biochem. 112, 1630-1642.

Gelmedin, V., Caballero-Gamiz, R., Brehm, K., 2008. Characterization and inhibition of a p38like mitogen-activated protein kinase (MAPK) from Echinococcus multilocularis: antiparasitic activities of p38 MAPK inhibitors. Biochem. Pharmacol. 76, 1068-1081.

Gelmedin, V., Spiliotis, M., Brehm, K., 2010. Molecular characterisation of MEK1/2- and MKK3/6-like mitogen-activated protein kinase kinases (MAPKK) from the fox tapeworm Echinococcus multilocularis. Int. J. Parasitol. 40, 555-567. 
Godot, V., Harraga, S., Beurton, I., Tiberghien, P., Sarciron, E., Gottstein, B., Vuitton, D.A., 2000. Resistance/susceptibility to Echinococcus multilocularis infection and cytokine profile in humans. II. Influence of the HLA B8, DR3, DQ2 haplotype. Clin. Exp. Immunol. $121,491-498$.

Gottstein, B., Felleisen, R., 1995. Protective immune mechanisms against the metacestode of Echinococcus multilocularis. Parasitol. Today 11, 320-326.

Gottstein, B., Wittwer, M., Schild, M., Merli, M., Leib, S.L., Müller, N., Müller, J., Jaggi, R., 2010. Hepatic gene expression profile in mice perorally infected with Echinococcus multilocularis eggs. PLoS One 5, e9779.

Hildreth, M.B., Granholm, N.H., 2003. Effect of mouse strain variations and cortisone treatment on the establishment and growth of primary Echinococcus multilocularis hydatid cysts. J. Parasitol. 89, 493-495.

Hillebrandt, S., Wasmuth, H.E., Weiskirchen, R., Hellerbrand, C., Keppeler, H., Werth, A., Schirin-Sokhan, R., Wilkens, G., Geier, A., Lorenzen, J., Köhl, J., Gressner, A.M., Matern, S., Lammert, F., 2005. Complement factor 5 is a quantitative trait gene that modifies liver fibrogenesis in mice and humans. Nat. Genet. 37, 835-843. 
Iraqi, F.A., Behnke, J.M., Menge, D.M., Lowe, A.M., Teale, A.J., Gibson, J.P., Baker, L.R., Wakelin, D.R., 2003. Chromosomal regions controlling resistance to gastro-intestinal nematode infections in mice. Mamm. Genome 14, 184-191.

Kassis, A.I., Tanner, C.E., 1977. Host serum proteins in Echinococcus multilocularis: complement activation via the classical pathway. Immunology 33, 1-9.

Konrad, C., Kroner, A., Spiliotis, M., Zavala-Góngora, R., Brehm, K., 2003. Identification and molecular characterisation of a gene encoding a member of the insulin receptor family in Echinococcus multilocularis. Int. J. Parasitol. 33, 301-312.

Korstanje, R., Eriksson, P., Samnegård, A., Olsson, P.G., Forsman-Semb, K., Sen, S., Churchill, G.A., Rollins, J., Harris, S., Hamsten, A., Paigen, B., 2004. Locating Ath8, a locus for murine atherosclerosis susceptibility and testing several of its candidate genes in mice and humans. Atherosclerosis 177, 443-450.

Lander, E., Kruglyak, L., 1995. Genetic dissection of complex traits: guidelines for interpreting and reporting linkage results. Nat. Genet. 11, 241-247.

Manly, K., Cudmore, R.J., Meer, J., 2001. Map Manager QTX, cross-platform software for genetic mapping. Mamm. Genome 12, 930-932.

Matsumoto, J., Kouguchi, H., Oku, Y., Yagi, K., 2010. Primary alveolar echinococcosis: course of larval development and antibody responses in intermediate host rodents with different 
genetic backgrounds after oral infection with eggs of Echinococcus multilocularis. Parasitol.

Int. 59, 435-444.

Nakaya, K., Nakao, M., Ito, A., 1997. Echinococcus multilocularis: mouse strain difference in hydatid development. J. Helminthol. 71, 53-56.

Peters, L.L., Robledo, R.F., Bult, C.J., Churchill, G.A., Paigen, B.J., Svenson, K.L., 2007. The mouse as a model for human biology: a resource guide for complex trait analysis. Nat. Rev. Genet. 8, 58-69.

Rennie, C., Hulme, H., Fisher, P., Halp, L., Agaba, M., Noyes, H.A., Kemp, S.J., Brass, A., 2008. A systematic, data-driven approach to the combined analysis of microarray and QTL data. Dev. Biol. (Basel) 132, 293-299.

Spiliotis, M., Konrad, C., Gelmedin, V., Tappe, D., Brückner, S., Mösch, H.U., Brehm, K., 2006. Characterisation of EmMPK1, an ERK-like MAP kinase from Echinococcus multilocularis which is activated in response to human epidermal growth factor. Int. J. Parasitol. 36, 10971112.

Spiliotis, M., Kroner, A., Brehm, K., 2003. Identification, molecular characterization and expression of the gene encoding the epidermal growth factor receptor orthologue from the fox-tapeworm Echinococcus multilocularis. Gene 323, 57-65. 
Spiliotis, M., Tappe, D., Brückner, S., Mösch, H.U., Brehm, K., 2005. Molecular cloning and characterization of Ras- and Raf-homologues from the fox-tapeworm Echinococcus multilocularis. Mol. Biochem. Parasitol. 139, 225-237.

Stark, J.M., Barmada, M.M., Winterberg, A.V., Majumber, N., Gibbons, W.J., Stark, M.A., Sartor, M.A., Medvedovic, M., Kolls, J., Bein, K., Mailaparambil, B., Krueger, M., Heinzmann, A., Leikauf, G.D., Prows, D.R., 2010. Genomewide association analysis of respiratory syncytial virus infection in mice. J. Virol. 84, 2257-2269.

Suzuki, T., Ishih, A., Kino, H., Muregi, F.W., Takabayashi, S., Nishikawa, T., Takagi, H., Terada, M., 2006. Chromosomal mapping of host resistance loci to Trichinella spiralis nematode infection in rats. Immunogenetics 58, 26-30.

Vuitton, D.A., 2003. The ambiguous role of immunity in echinococcosis: protection of the host or of the parasite? Acta Trop. 85, 119-132.

Vuitton, D.A., Gottstein, B., 2010. Echinococcus multilocularis and its intermediate host: a model of parasite-host interplay. J. Biomed. Biotechnol. 2010, 923193.

Wayne, M.L., McIntyre, L.M., 2002. Combining mapping and arraying: An approach to candidate gene identification. Proc. Natl. Acad. Sci. USA 99, 14903-14906.

Webster, G.A., Cameron, T.W.M., 1961. Observations on experimental infections with Echinococcus in rodents. Can. J. Zool. 39, 877-891. 
Williams-Blangero, S., Vandeberg, J.L., Subedi, J., Jha, B., Dyer, T.D., Blangero, J., 2008. Two quantitative trait loci influence whipworm (Trichuris trichiura) infection in a Nepalese population. J. Infect. Dis. 197, 1198-1203.

Yamashita, J., Ohbayashi, M., Kitamura, Y., Suzuki, K., Okugi, M., 1958. Studies on echinococcosis VIII. Experimental Echinococcosis multilocularis in various rodents; especially on the difference of susceptibility among uniform strains of the mouse. Jpn. J. Vet. Res. 6, 135-159.

Yang, Y.R., Ellis, M., Sun, T., Li, Z., Liu, X., Vuitton, D.A., Bartholomot, B., Giraudoux, P., Craig, P.S., Boufana, B., Wang, Y., Feng, X., Wen, H., Ito, A., McManus, D.P., 2006. Unique family clustering of human echinococcosis cases in a chinese community. Am. J. Trop. Med. Hyg. 74, 487-494.

Yoshida, Y., Makita, Y., Heida, N., Asano, S., Matsushima, A., Ishii, M., Mochizuki, Y., Masuya, H., Wakana, S., Kobayashi, N., Toyoda, T., 2009. PosMed (Positional Medline): prioritizing genes with an artificial neural network comprising medical documents to accelerate positional cloning. Nucleic Acids Res. 37, W147-152.

Zavala-Góngora, R., Derrer, B., Gelmedin, V., Knaus, P., Brehm, K., 2008. Molecular characterisation of a second structurally unusual AR-Smad without an MH1 domain and a Smad4 orthologue from Echinococcus multilocularis. Int. J. Parasitol. 38, 161-176. 
Zavala-Góngora, R., Kroner, A., Bernthaler, P., Knaus, P., Brehm, K., 2006. A member of the transforming growth factor-beta receptor family from Echinococcus multilocularis is activated by human bone morphogenetic protein 2. Mol. Biochem. Parasitol. 146, 265-271.

Zavala-Góngora, R., Kroner, A., Wittek, B., Knaus, P., Brehm, K., 2003. Identification and characterisation of two distinct Smad proteins from the fox-tapeworm Echinococcus multilocularis. Int. J. Parasitol. 33, 1665-1677.

Zhang, S., Penfornis, A., Harraga, S., Chabod, J., Beurton, I., Bresson-Hadni, S., Tiberghien, P., Kern, P., Vuitton, D.A., 2003. Polymorphisms of the TAP1 and TAP2 genes in human alveolar echinococcosis. Eur. J. Immunogenet. 30, 133-139. 
Table 1. List of microsatellite markers used for whole genome scanning in this study.

\begin{tabular}{|c|c|c|c|c|c|c|c|c|c|c|c|}
\hline Markers & $\mathrm{cM}$ & CYS & PSC & Markers & $\mathrm{cM}$ & CYS & PSC & Markers & $\mathrm{cM}$ & CYS & PSC \\
\hline D1Mit118 & 1.65 & + & + & D6Mit159 & 12.36 & + & + & D13Mit17 & 7.73 & + & + \\
\hline D1Mit324 & 29.13 & + & + & D6Mit74 & 23.70 & + & + & D13Mit60 & 14.44 & + & + \\
\hline D1Mit415 & 43.94 & + & + & D6Mit188 & 32.53 & + & + & D13Mit63 & 21.00 & + & + \\
\hline D1Mit191 & 52.66 & + & + & D6Mit104 & 51.53 & + & & D13Mit9 & 42.19 & + & + \\
\hline D1Mit30 & 57.91 & + & + & D6Mit150 & 53.75 & + & + & D13Mit148 & 59.69 & + & + \\
\hline D1Mit445 & 58.27 & & + & D6Mit254 & 59.32 & + & & D13Mit262 & 63.93 & + & + \\
\hline D1Mit14 & 67.71 & + & + & D6Mit374 & 64.60 & + & & & & & \\
\hline D1Mit145 & 74.68 & & + & D6Mit59 & 69.36 & + & & D14Mit10 & 6.41 & + & + \\
\hline D1Mit355 & 80.36 & & + & D6Mit15 & 77.70 & + & + & D14Mit120 & 20.88 & + & + \\
\hline D1Mit150 & 81.08 & & + & & & & & D14Mit102 & 34.36 & + & + \\
\hline D1Mit291 & 88.97 & + & + & D7Mit114 & 15.42 & + & + & D14Mit225 & 39.46 & + & + \\
\hline \multirow[t]{2}{*}{ D1Mit511 } & 93.70 & & + & D7Mit82 & 32.76 & + & + & D14Mit165 & 56.16 & + & + \\
\hline & & & & D7Mit318 & 42.27 & + & + & D14Mit266 & 64.86 & + & + \\
\hline D2Mit293 & 17.24 & + & + & D7Mit66 & 64.30 & + & + & & & & \\
\hline D2Mit296 & 21.81 & + & + & D7Mit333 & 82.25 & + & + & D15Mit12 & 1.80 & + & + \\
\hline D2Mit91 & 39.24 & + & + & & & & & D15Mit5 & 16.74 & + & + \\
\hline D2Mit185 & 55.23 & + & + & D8Mit4 & 18.89 & + & + & D15Mit156 & 32.19 & + & + \\
\hline D2Mit62 & 59.34 & + & + & D8Mit100 & 29.70 & + & + & D15Mit159 & 41.96 & + & + \\
\hline D2Mit286 & 76.74 & + & + & D8Mit234 & 39.33 & + & + & D15Mit161 & 52.78 & + & + \\
\hline D2Mit 229 & 88.99 & + & + & D8Mit242 & 50.07 & + & + & & & & \\
\hline \multirow{2}{*}{ D2Mit200 } & 102.29 & + & + & D8Mit200 & 61.37 & + & + & D16Mit182 & 2.57 & + & + \\
\hline & & & & & & & & D16Mit59 & 26.86 & + & + \\
\hline D3Mit164 & 2.01 & + & + & D9Mit90 & 17.80 & + & + & D16Mit140 & 40.30 & + & + \\
\hline D3Mit182 & 21.73 & + & + & D9Mit91 & 20.74 & + & + & D16Mit152 & 48.23 & + & + \\
\hline D3Mit28 & 39.27 & + & + & D9Mit302 & 36.36 & + & + & D16Mit106 & 57.68 & + & + \\
\hline D3Mit14 & 61.32 & + & + & D9Mit133 & 45.80 & + & + & & & & \\
\hline \multirow[t]{2}{*}{ D3Mit129 } & 80.49 & + & + & D9Mit355 & 51.41 & + & + & D17Mit113 & 8.14 & + & + \\
\hline & & & & D9Mit18 & 71.49 & + & + & D17Mit198 & 14.59 & & + \\
\hline D4Mit 235 & 3.57 & + & + & & & & & D17Mit139 & 27.40 & & + \\
\hline D4Mit $237 a$ & 22.38 & + & + & D10Mit248 & 5.21 & + & + & D17Mit89 & 32.82 & + & + \\
\hline D4Mit139 & 29.65 & + & + & D10Mit61 & 34.80 & + & + & D17Mit218 & 43.76 & & + \\
\hline D4Mit152 & 39.46 & + & + & D10Mit186 & 38.56 & + & + & D17Mit187 & 50.17 & + & + \\
\hline D4Mit303 & 45.55 & + & + & D10Mit14 & 66.75 & + & + & D17Mit221 & 59.77 & + & + \\
\hline D4Mit308 & 57.66 & + & + & D10Mit297 & 72.31 & + & + & & & & \\
\hline D4Mit54 & 70.02 & + & + & & & & & D18Mit132 & 11.92 & + & + \\
\hline \multirow[t]{2}{*}{ D4Mit42 } & 82.64 & + & + & D11Mit226 & 5.64 & + & + & D18Mit17 & 21.09 & + & + \\
\hline & & & & D11Mit21 & 25.94 & + & + & D18Mit124 & 32.15 & + & + \\
\hline D5Mit180 & 11.93 & + & + & D11Mit140 & 32.13 & + & + & D18Mit184 & 39.70 & + & + \\
\hline D5Mit108 & 23.91 & + & + & Dl1Mit4 & 41.87 & + & + & D18Mit7 & 51.92 & + & + \\
\hline D5Mit258 & 33.95 & + & + & D11Mit212 & 54.34 & + & + & & & & \\
\hline D5Mit208 & 48.51 & + & + & D11Mit199 & 65.48 & + & + & D19Mit69 & 8.93 & + & + \\
\hline D5Mit 188 & 57.51 & + & + & D11Mit48 & 82.96 & + & + & D19Mit80 & 18.24 & + & + \\
\hline D5Mit370 & 65.23 & + & + & & & & & D19Mit33 & 51.76 & + & + \\
\hline \multirow[t]{4}{*}{ D5Mit222 } & 81.53 & + & + & D12Mit 219 & 9.69 & + & + & & & & \\
\hline & & & & D12Mit172 & 21.09 & + & + & DXMit166 & 28.26 & + & + \\
\hline & & & & D12Mit5 & 37.16 & + & + & DXMit130 & 55.45 & + & + \\
\hline & & & & D12Mit101 & 51.55 & + & + & DXMit186 & 76.75 & + & + \\
\hline
\end{tabular}

cM, centiMorgan; CYS, markers used for Quantitative trait loci (QTLs) analysis of cyst establishment; PSC, markers used for QTL analysis of protoscolex development. 
Table 2. Quantitative trait loci (QTLs) with high likelihood ratio statistic (LRS) scores (> 4.0) detected by marker regression analysis.

\begin{tabular}{|c|c|c|c|c|c|c|}
\hline Chr. & Locus & LRS & Contribution (\%) & $P$ value & $\mathrm{CI}$ & Additive effect \\
\hline \multicolumn{7}{|c|}{ Cyst establishment } \\
\hline 3 & D3Mit164 & 4.8 & 4 & 0.02847 & 113 & -14.07 \\
\hline 6 & D6Mit104 & 13.1 & 10 & 0.00030 & 43 & -22.72 \\
\hline 6 & D6Mit150 & 15.0 & 12 & 0.00011 & 38 & -24.32 \\
\hline 6 & D6Mit254 & 14.7 & 12 & 0.00013 & 38 & -24.05 \\
\hline 6 & D6Mit374 & 11.0 & 9 & 0.00092 & 51 & -20.95 \\
\hline 6 & D6Mit59 & 7.2 & 6 & 0.00724 & 76 & -17.14 \\
\hline 6 & D6Mit15 & 4.3 & 4 & 0.03725 & 124 & -13.37 \\
\hline 9 & D9Mit91 & 6.7 & 5 & 0.00989 & 82 & 16.44 \\
\hline 13 & D13Mit9 & 5.7 & 5 & 0.01724 & 96 & -15.21 \\
\hline 13 & D13Mit148 & 5.3 & 4 & 0.02152 & 103 & -14.73 \\
\hline 13 & D13Mit262 & 5.2 & 4 & 0.02211 & 103 & -14.63 \\
\hline 17 & D17Mit187 & 4.5 & 4 & 0.03457 & 121 & 13.55 \\
\hline 17 & D17Mit221 & 4.0 & 3 & 0.04627 & 136 & 12.78 \\
\hline \multicolumn{7}{|c|}{ Protoscolex development } \\
\hline 1 & D1Mit415 & 7.2 & 6 & 0.00734 & 76 & -0.75 \\
\hline 1 & D1Mit191 & 17.9 & 15 & 0.00002 & 32 & -1.15 \\
\hline 1 & D1Mit30 & 31.4 & 24 & 0.00000 & 19 & -1.48 \\
\hline 1 & D1Mit445 & 31.4 & 24 & 0.00000 & 19 & -1.48 \\
\hline 1 & D1Mit14 & 75.4 & 48 & 0.00000 & 10 & -2.12 \\
\hline 1 & D1Mit145 & 73.5 & 48 & 0.00000 & 10 & -2.09 \\
\hline 1 & D1Mit355 & 42.5 & 31 & 0.00000 & 15 & -1.70 \\
\hline 1 & D1Mit150 & 40.4 & 30 & 0.00000 & 16 & -1.68 \\
\hline 1 & D1Mit291 & 29.5 & 23 & 0.00000 & 20 & -1.46 \\
\hline 1 & D1Mit511 & 18.5 & 15 & 0.00002 & 31 & -1.18 \\
\hline 2 & D2Mit296 & 4.2 & 4 & 0.03958 & 127 & -0.58 \\
\hline 3 & D3Mit182 & 4.4 & 4 & 0.03551 & 122 & 0.59 \\
\hline 5 & D5Mit222 & 5.4 & 5 & 0.02063 & 101 & -0.67 \\
\hline 6 & D6Mit188 & 7.1 & 6 & 0.00765 & 77 & 0.74 \\
\hline 14 & D14Mit165 & 6.2 & 5 & 0.01274 & 88 & -0.71 \\
\hline 17 & D17Mit198 & 4.5 & 4 & 0.03307 & 119 & 0.60 \\
\hline 17 & D17Mit139 & 7.3 & 6 & 0.00679 & 75 & 0.76 \\
\hline 17 & D17Mit89 & 5.6 & 5 & 0.01809 & 97 & 0.66 \\
\hline 17 & D17Mit218 & 9.2 & 8 & 0.00247 & 60 & 0.85 \\
\hline 17 & D17Mit187 & 10.3 & 9 & 0.00130 & 54 & 0.89 \\
\hline 19 & D19Mit69 & 6.0 & 5 & 0.01397 & 90 & -0.69 \\
\hline$X$ & DXMit186 & 5.9 & 5 & 0.01557 & 93 & 0.70 \\
\hline
\end{tabular}

Chr., chromosome; CI, confidence interval. 
Table 3. List of candidate genes for Emcys1 and Empsc1 of Mus musculus.

\begin{tabular}{|c|c|c|}
\hline Gene symbol & Gene function & $\mathrm{cM}$ \\
\hline \multicolumn{3}{|c|}{ Emcys 1 candidates on Chr. 6} \\
\hline D6Mit188 & Flanking marker & 32.53 \\
\hline $\mathrm{Cd} 207$ & CD 207 antigen & 35.94 \\
\hline Trh & Thyrotropin releasing hormone & 41.03 \\
\hline Il5ra & Interleukin 5 receptor, alpha & 49.19 \\
\hline Vhlh & Von Hippel-Lindau syndrome homolog & 52.81 \\
\hline Ghrl & Ghrelin & 52.84 \\
\hline $\operatorname{Hrhl}$ & Histamine receptor $\mathrm{H} 1$ & 53.05 \\
\hline D6Mit150 & & 53.75 \\
\hline $8-M a r$ & Membrane-associated ring finger $(\mathrm{C} 3 \mathrm{HC} 4) 8$ & 53.78 \\
\hline Cxcl12 & Chemokine (C-X-C motif) ligand 12 & 54.81 \\
\hline C3arl & Complement component 3 a receptor 1 & 57.93 \\
\hline Cd163 & CD163 antigen & 59.13 \\
\hline$C 1 r l$ & Complement component $1, \mathrm{r}$ subcomponent-like & 59.16 \\
\hline$C 1 r$ & Complement component $1, \mathrm{r}$ subcomponent & 59.16 \\
\hline$C 1 s$ & Complement component 1 , s subcomponent & 59.17 \\
\hline$C d 4$ & CD4 antigen & 59.17 \\
\hline $\operatorname{Lag} 3$ & Lymphocyte-activation gene 3 & 59.17 \\
\hline Tnfrsfla & Tumor necrosis factor receptor superfamily, member 1a & 59.32 \\
\hline D6Mit254 & & 59.32 \\
\hline Fgf 23 & Fibroblast growth factor 23 & 61.92 \\
\hline Klrblc & Killer cell lectin-like receptor subfamily B member 1C & 63.09 \\
\hline Cd69 & CD69 antigen & 63.21 \\
\hline Clec $7 a$ & C-type lectin domain family 7 , member a & 63.34 \\
\hline D6Mit15 & Flanking marker & 77.70 \\
\hline \multicolumn{3}{|c|}{ Empscl candidates on Chr. 1} \\
\hline D1Mit445 & Flanking marker & 58.27 \\
\hline Ptprc & Protein tyrosine phosphatase, receptor type, C & 60.73 \\
\hline $\operatorname{Rgs} 1,2,13$ & Regulator of G-protein signaling $1,2,13$ & 62.56 \\
\hline $\operatorname{Rgs} 18$ & Regulator of G-protein signaling 18 & 62.99 \\
\hline Pla $2 g 4 a$ & Phospholipase A2, group IVA (cytosolic, calcium-dependent) & 63.51 \\
\hline Ptgs 2 & Prostaglandin-endoperoxide synthase 2 & 63.84 \\
\hline $\operatorname{Rgs} 8$ & Regulator of G-protein signaling 8 & 65.41 \\
\hline Rgsll, 2 & Regulator of G-protein signaling like 1,2 & 65.43 \\
\hline Rgs16 & Regulator of G-protein signaling 16 & 65.43 \\
\hline$A b l 2$ & V-abl Abelson murine leukemia viral oncogene 2 (arg, Abelson-related gene) & 67.71 \\
\hline D1Mit14 & & 67.71 \\
\hline Fasl & Fas ligand (TNF superfamily, member 6) & 69.95 \\
\hline Sele & Selectin, endothelial cell & 71.35 \\
\hline Sell & Selectin, lymphocyte & 71.37 \\
\hline Selp & Selectin, platelet & 71.42 \\
\hline Tiprl & TIP41, TOR signalling pathway regulator-like (S. cerevisiae) & 72.55 \\
\hline $\operatorname{Rgs} 4,5$ & Regulator of G-protein signaling 4, 5 & 76.84 \\
\hline Atf6 & Activating transcription factor 6 & 76.96 \\
\hline Fcgr $2 b$ & Fc receptor, IgG, low affinity Iib & 78.02 \\
\hline Fcgr3 & Fc receptor, IgG, low affinity III & 78.80 \\
\hline Fcerlg & Fc receptor, IgE, high affinity I, gamma polypeptide & 79.23 \\
\hline Slamf1 & Signaling lymphocytic activation molecule family member 1 & 79.54 \\
\hline$C d 48$ & CD48 antigen & 79.54 \\
\hline Ncstn & Nicastrin & 79.54 \\
\hline $\mathrm{Crp}$ & C-reactive protein, petaxin related & 80.13 \\
\hline Fcerla & Fc receptor, IgE, high affinity I, alpha polypeptide & 80.33 \\
\hline D1Mit355 & Flanking marker & 80.36 \\
\hline
\end{tabular}

cM, centiMorgan; Chr., chromosome. 
Fig. 1. Distribution of cyst establishment (A) and protoscolex development (B) of Echinococcus multilocularis in the livers of D2, B6, $\mathrm{F}_{1}$ and $\mathrm{N}_{2}$ mice.

A

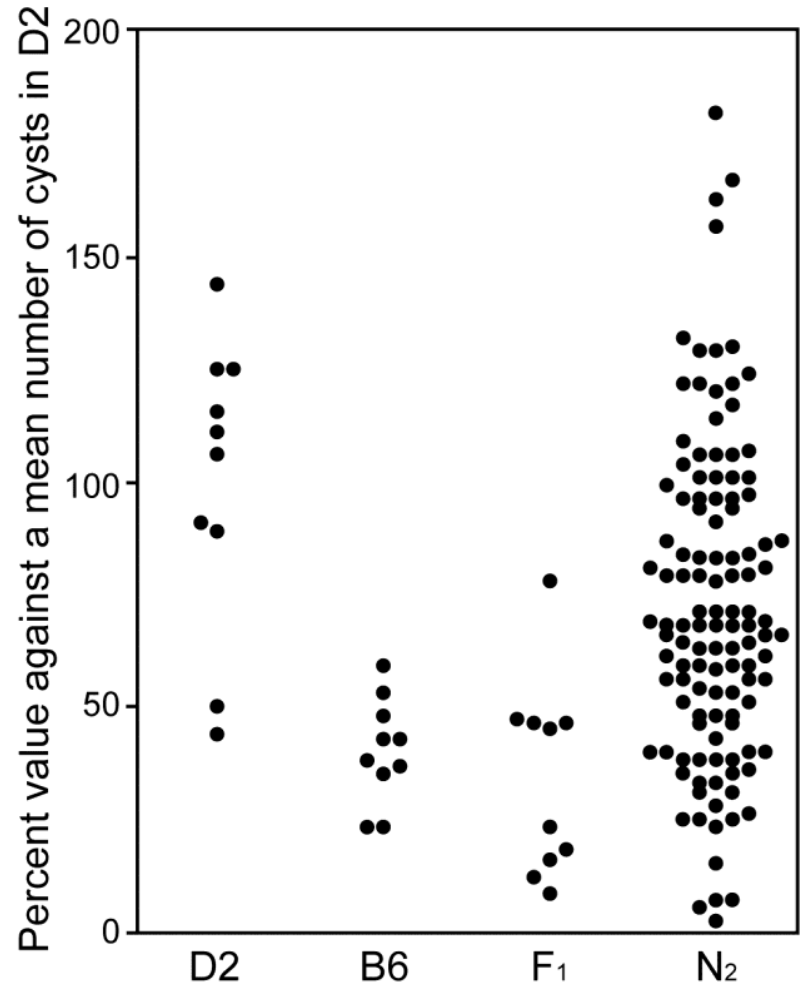

B

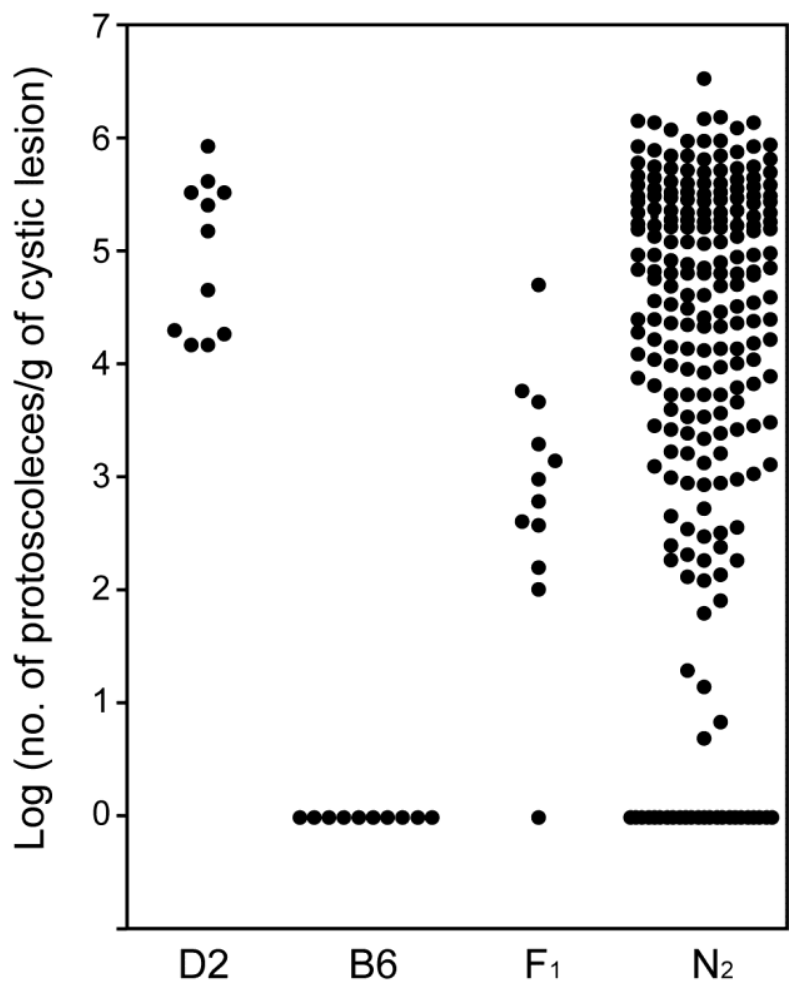

(A) The number of cysts in the liver was counted 4 weeks p.i. with 200 eggs for the assessment of cyst establishment. The numbers were converted to percent values against a mean number of cysts in susceptible D2 mice. (B) The number of protoscoleces was counted 16 weeks p.i. with 2,000 eggs for the assessment of protoscolex development. The numbers were indicated as number/gram of cystic lesion and subsequently log-transformed. Zero values were assigned a value of 1 to allow log transformation. 
Fig. 2. Whole genome scans for chromosome sites associated with cyst establishment (A) and protoscolex development (B) of Echinococcus multilocularis in the liver of $\mathrm{N}_{2}$ mice.

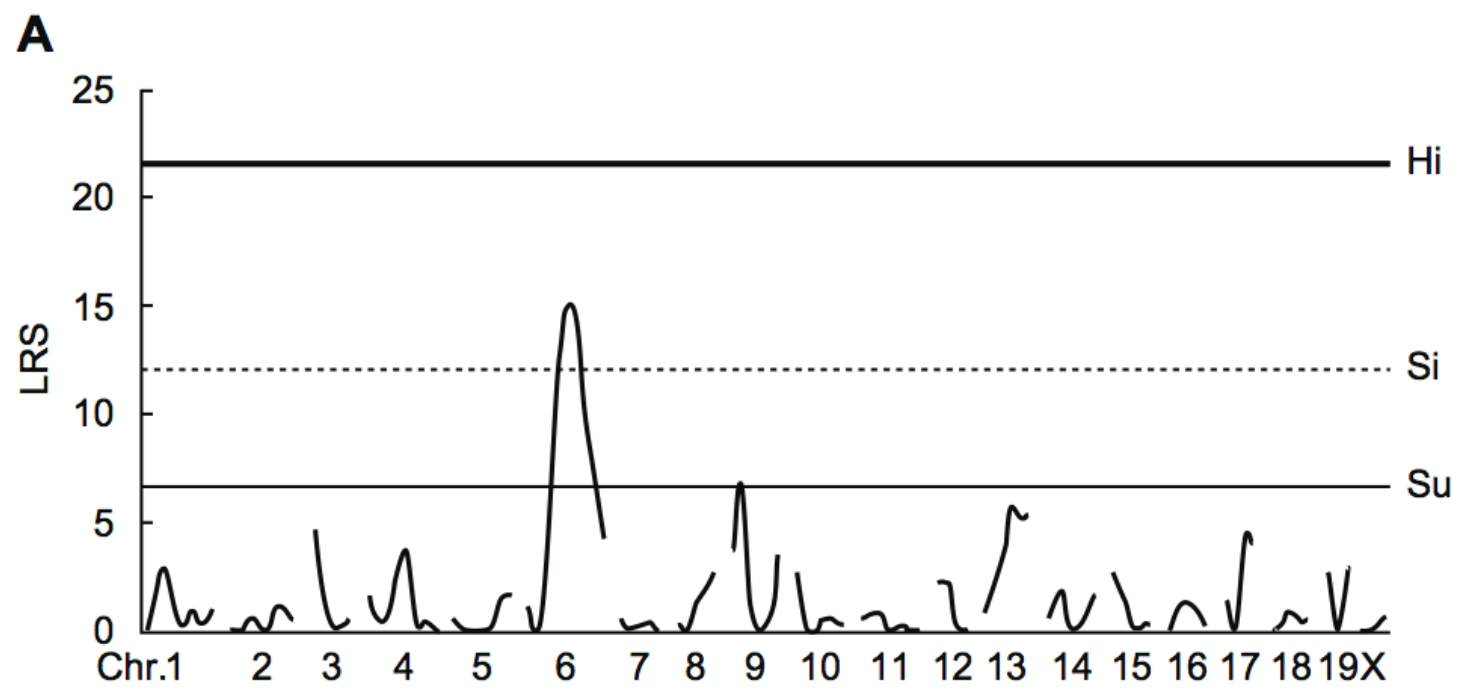

B

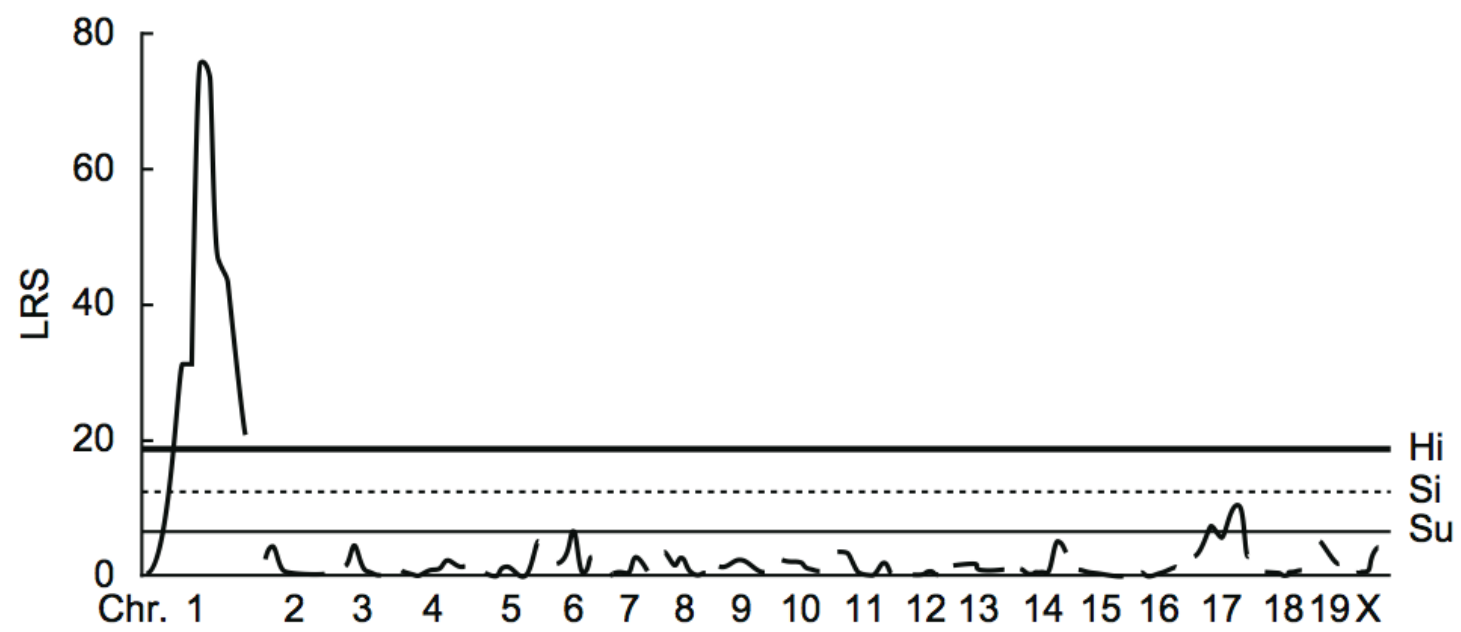

Analyses of linkage of cyst establishment (A) and protoscolex development (B) in the liver of $\mathrm{N}_{2}$ mice to chromosomal loci were performed using the MapManager QTXb20 software. Recombination frequencies (\%) were converted into genetic distance (centiMorgan; cM) using the Kosambi map function, in which linkage data are provided as likelihood ratio statistic (LRS) scores. Genome-wide significance thresholds were calculated in terms of LRS by carrying out permutation tests for 1,000 permutations. The thresholds for suggestive ( $\mathrm{Su}$ ), significant ( $\mathrm{Si}$ ) and highly significant (Hi) linkages are indicated in thin, dotted and thick lines, respectively. 
Fig. 3. Quantitative trait loci (QTLs) affecting cyst establishment (A) and protoscolex development (B) of Echinococcus multilocularis in the liver.

A

Chr. 6

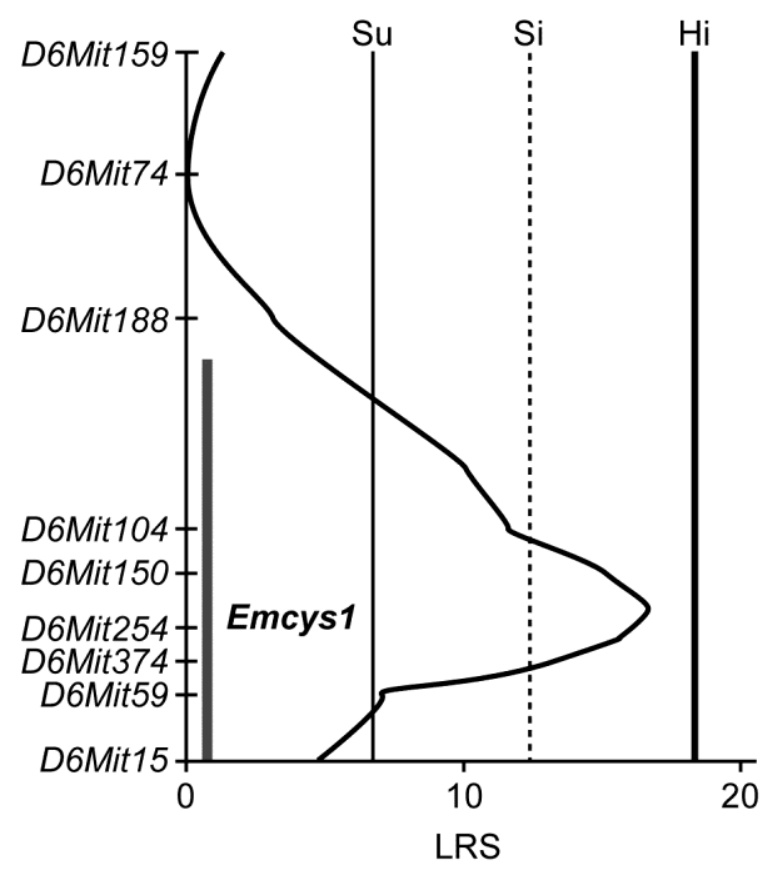

B

Chr. 1

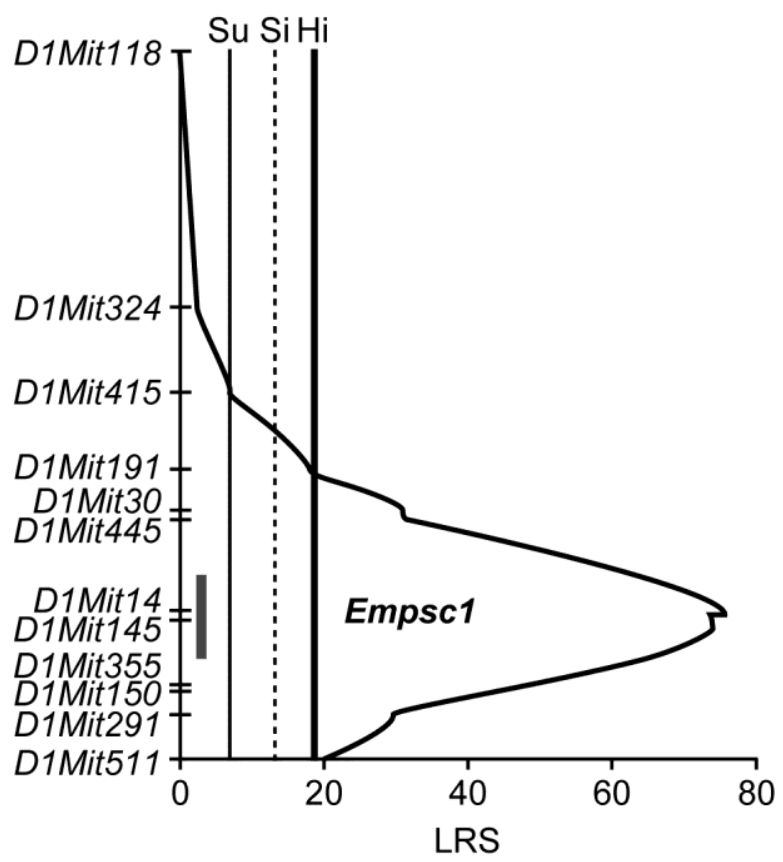

The QTLs on chromosomes (Chrs.) 6 (Emcys1) and 1 (Empsc1) showed significant and highly significant linkage to (A) cyst establishment and (B) protoscolex development, respectively. The gray bars on the graph indicate approximate $95 \%$ confidence intervals. The thin, dotted and thick lines represent suggestive $(\mathrm{Su})$, significant $(\mathrm{Si})$ and highly significant $(\mathrm{Hi})$ thresholds calculated by 1,000 times permutation tests, respectively. The microsatellite markers used for determining genotypes of $\mathrm{N}_{2}$ mice are presented along the Y-axis. LRS, likelihood ratio statistic. 
Fig. 4. Effect of allelic combination at D6Mit150 (A) and D1Mit14 (B) on $\mathrm{N}_{2}$ mice.

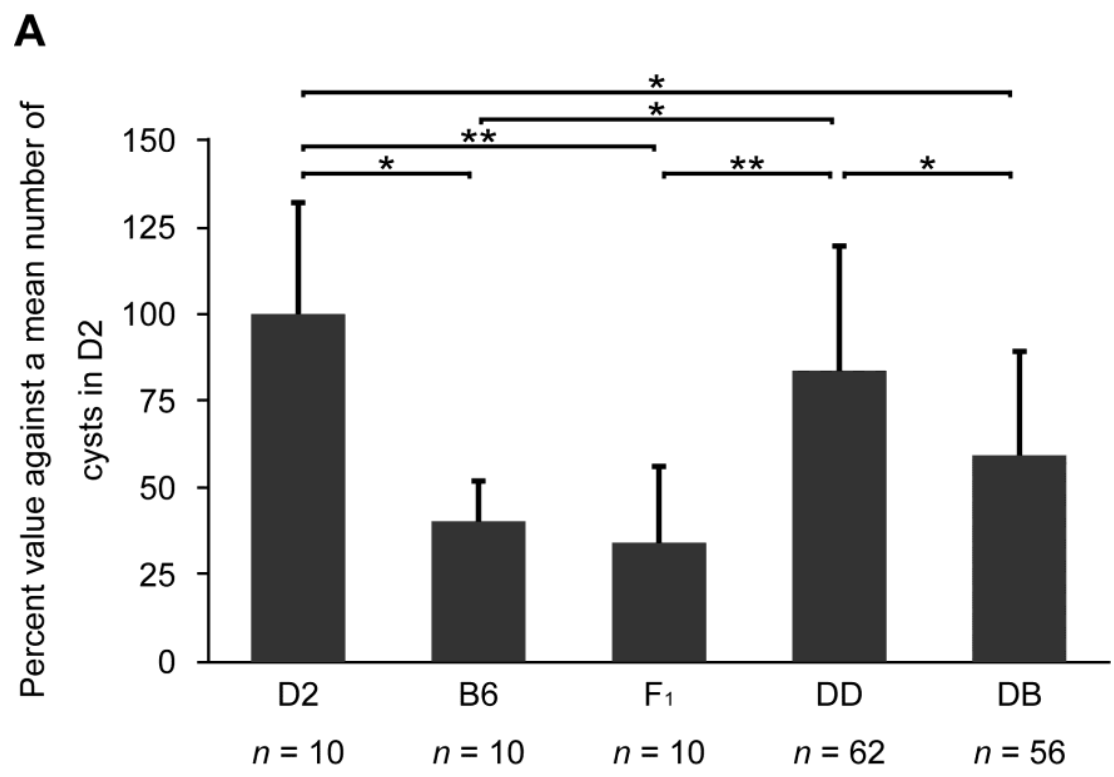

B

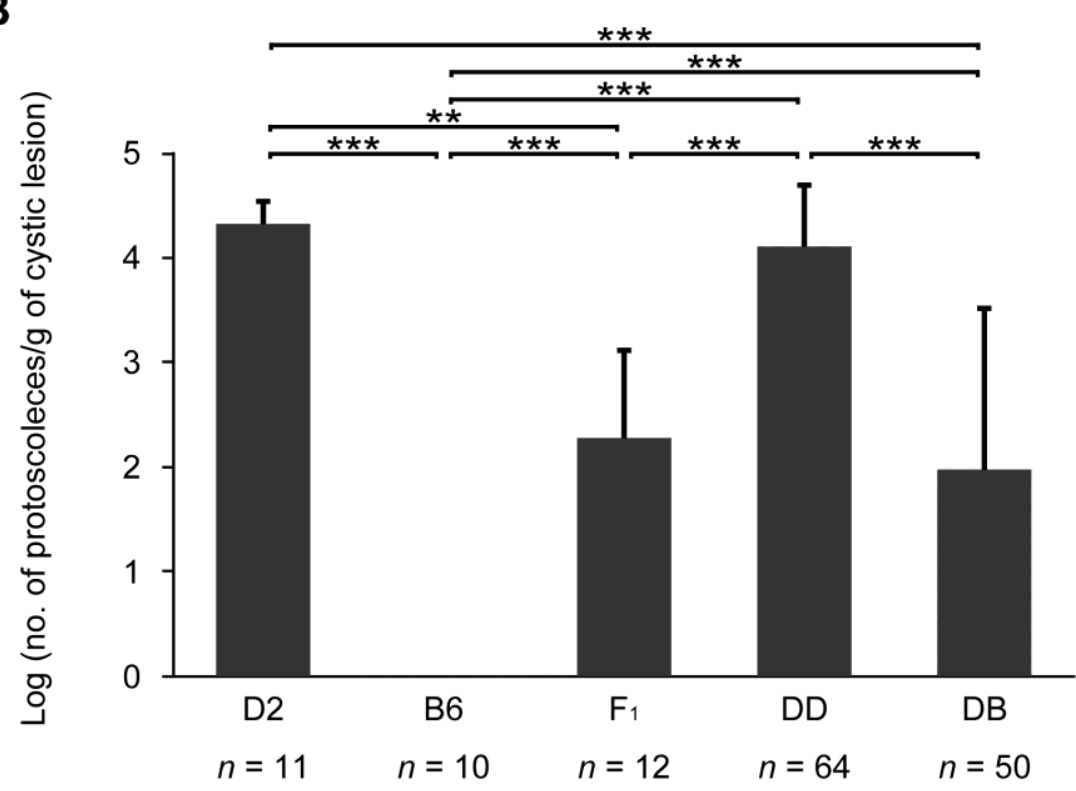

$\mathrm{N}_{2}$ mice were segregated according to their respective genotypes at (A) D6Mit150, a marker representing the Emcysl locus and (B) DIMit14, a marker representing the Empscl locus. The mean value of each group was shown with the bar representing the S.D.. DD and DB indicate individuals homozygous for the D2 allele and heterozygous for D2 and B6 alleles, respectively. The number of animals in each group is indicated at the bottom. Statistical analysis for multiple comparisons between the mean values of each group was conducted using Scheffé 's F test $(* P<$ $0.05 ; * * P<0.001 ; * * * P<0.0001)$. 